\title{
Manchette des paumés ou la marginalité intérieure des personnages du roi du néo-polar
}

\author{
Marcela Poučová \\ Université Masaryk \\ poucova@ped.muni.cz
}

Rebut: 2 de maig de 2018

Acceptat: 7 de juliol de 2018

\section{RESUM}

Manchette des paumés o la marginalitat interna dels personatges del rei del neopolar

La història del neopolar francès és sobretot una història de protesta contra el conformisme i la pertinença a les estructures oficials de la societat. El disgust cap a la societat de consum manté als personatges del neopolar en una marginalitat interna que, quan es produeix una situació de crisi, aflora a la superfície i els personatges - ja víctimes dels seus propis fantasmes- es converteixen en víctimes dels representants de l'ordre públic. El lliscament lent o brusc de la marginalitat interna igual que l'externa és el tema de la nostra anàlisi que es basa en les novel-les Nada (1973), Le petit Bleu de la côte ouest (1976) i La Position du tireur couché (1981) de l'autor emblemàtic del neopolar Jean-Patrick Manchette (1942-1995).

\section{Paraules Clau}

Novel-la detectivesca francesa, novel-la negra, terrorisme, història de França 1970-1980, marginalitat, Jean-Patrick Manchette.

\section{RESUMEN}

\section{Manchette des paumés o la marginalidad interior de los personajes del rey} del neopolar

La historia del neopolar francés es sobre todo una historia de la protesta contra el conformismo y a la pertenencia a las estructuras oficiales de la sociedad. El disgusto hacia la sociedad de consumo mantiene a los personajes del neopolar en una marginalidad interior que, en la situación de crisis, llega a la superficie 
y los personajes - ya víctimas de sus propios fantasmas- se convierten en presas de los representantes de la orden pública. El deslizamiento lento o brutal de la marginalidad interior igual que la del exterior es el tema de nuestra análisis que se cedntra en las novelas Nada (1973), Balada de la costa Oeste (1976) y Caza al asesino (1981) del autor emblemático del neopolar JeanPatrick Manchette (1942-1995).

Palabras Clave

Novela detectivesca francesa, novela negra, terrorismo, historia de Francia 1970-1980, marginalidad, Jean-Patrick Manchette.

\section{RÉSUMÉ}

Manchette des paumés ou la marginalité intérieure des personnages du roi du néo-polar

L'histoire du néo-polar français est avant tout l'histoire d'une contestation du conformisme et de l'appartenance à des structures officielles de la société. Le dégout de la société de consommation maintient les personnages des néopolars dans une marginalité intérieure qui, dans une situation de crise, remonte à la surface et les personnages - déjà en proie à leurs propres fantasmes deviennent les proies des représentants de l'ordre public. Le glissement lent ou brutal de la marginalité intérieure et celle de l'extérieur est le sujet de notre analyse qui s'appuie sur les romans Nada (1973), Le petit Bleu de la côte ouest (1976) et La Position du tireur couché (1981) de l'auteur phare du néo-polar Jean-Patrick Manchette (1942-1995).

Mots CLÉS

Roman policier français, roman noir, terrorisme, France 1970-1980, marginalité, Jean-Patrick Manchette.

\section{AbSTRACT}

Manchette des paumés - On the inner marginality of the characters created by the king of the neo-polar genre

The story of the French neo-polar style is above all a story of disputing the acts of conforming and belonging to official structures of society. The disrespect the consumer society holds for the characters of the neo-polar genre places them in an internal marginality which, in a crisis situation, rises up to the surface and the characters - who are already prey to their own fantasies - become the prey of the representatives of the public order. Both the slow and abrupt shifts in inner and outer marginalization are the subject of our analysis which is based on the novels Nada (1973), The Little Blue of the West Coast (1976) and The 
Prone Gunman (1981) by the influential author of the neo-polar genre - JeanPatrick Manchette (1942-1995).

\section{KEY WORDS}

French crime fiction, Roman noir, Terrorism, French History 1970-1980, Marginality, Jean-Patrick Manchette.

L'histoire du néo-polar français est avant tout l'histoire d'une contestation du conformisme et de l'appartenance à des structures officielles de la société. La révolte contre l'establishment signifie pour les auteurs tout comme pour leurs personnages la révolte contre un status quo social révolu et insatisfaisant. Le gouvernement exerce selon eux, tout comme dans le monde dystopique d'Huxley, son pouvoir à l'aide d'un abêtissement général des masses reposant sur l'abondance de biens matériels. Le dégout de la société de consommation maintient les personnages (nous ne nous permettrons pas d'en dire autant sur les auteurs) dans une marginalité intérieure qui, dans une situation de crise, remonte à la surface et les personnages - déjà en proie à leurs propres fantasmes - deviennent les proies des représentants de l'ordre public. Le glissement lent ou brutal de la marginalité intérieure et celle de l'extérieur est le sujet de notre analyse qui s'appuie surtout sur les romans Nada (1973), Le petit Bleu de la côte ouest (1976) et La Position du tireur couché (1981) de l'auteur phare du néo-polar Jean-Patrick Manchette (1942-1995).

Jean-Patrick Manchette (1942-1995) entre dans la littérature en 1971. Un enfant doué, issu du milieu prolétaire, il montre depuis sa petite enfance un penchant pour la littérature, le cinéma, la musique et la politique. Une telle combinaison de goûts le conduit à son adolescence vers l'idéologie d'ultragauche plus précisément du mouvement situationniste. L'internationale situationniste (1957-1972) veut tout d'abord révolutionner l'art pour essayer ensuite de révolutionner la société de consommation qui s'instaure en Europe à partir des années 1930 et se massifie en France pendant les Trente Glorieuses (1945-1975). Manchette quant à lui trouve son champ de bataille dans le roman policier et noir qui se convertit sous son influence et passe d'une littérature passe-temps à une arme de la critique sociale. L'auteur de dix romans, de plusieurs scénarios (cinéma et télévision) et d'une innombrable quantité d'articles sur la thématique, remanie le paysage du roman policier et noir français à jamais. 
Le dictionnaire Larousse définie la marginalité comme «position marginale par rapport à une norme sociale. $»^{1}$ Si nous étendons cette définition à « une norme culturelle », la production littéraire situationniste de Manchette présente «plusieurs symptômes de la marginalité » qui se complètent, s'appuient et s'expliquent mutuellement. On peut y distinguer alors la marginalité formelle (le genre policier/noir); la marginalité idéologique (de l'extrême gauche) et la marginalité d'écriture (le style, le sujet et les personnages). Sur l'exemple des romans proposés, nous essayerons de les nommer et analyser dans leur ensemble.

En 1979, Manchette 《lecteur boulimique et éclectique $»^{2}$ et grand connaisseur de la culture américaine écrit :

Le polar classique a des bornes historiques (années 20, années 50), il a aussi un centre géographique, les États-Unis, centre du monde de ce temps, centre du marché mondial, centre de la contre-révolutionqui produit le polar. Quand l'art est devenu une marchandise (nommée culture), l'hégémonie économique et politique est aussi hégémonie culturelle. Face à l'hégémonie américaine, des formes anciennes peuvent survivre en se figeant [...], mais ce qui se crée de nouveau se définit nécessairement par son rapport aux formes américaines. Rapport d'imitation et de négation. ${ }^{3}$

Laissons de côté le caractère idéologique de cette définition pour se concentrer sur la définition que Manchette donne au polar. Il le nomme « cette petite forme sous-littéraire que le triomphe en question [la contre-révolution] a produite. ${ }^{4}$ Et c'est exactement cette littérature mineure, cette forme sous ou para littéraire bien à la marge du champ littéraire qui charme Manchette.

Manchette publie son premier polar en collaboration avec Jean-Pierre Bastide en $1971^{5}$ et le succès ne se fait pas attendre. Bien qu'il y ait d'autres auteurs qui flairent le changement du registre narratif du genre, c'est Manchette

\footnotetext{
1 "Marginalité". (2018, 7 avril). Dictionnaires Larousse. Page consultée le $13: 25$, avril 7, 2018 à partir de https://www.larousse.fr/dictionnaires/francais/marginalit\%C3\%A9/49449\#VeHjZZWYp 8F5MXlf.99.

${ }^{2}$ Charybde 27, Je me souviens de : «Nada» (Jean-Patrick Manchette). (2018, avril 6) Page consultée le $8:$ 15, avril 6, 2018 à partir de https://charybde2.wordpress.com/2017/05/06/je-mesouviens-de-nada-jean-patrick-manchette/.

${ }^{3}$ Jean-Patrick MANCHETTE, Chroniques, Payot \& Rivages, Paris, 2013, p. 85.

${ }^{4}$ Ibidem p. 86.

${ }^{5}$ Il s'agit du roman Laissez bronzer les cadavres. Il attend jusqu'en 2017 pour d'être porté à l'écran par Hélène Cattet et Bruno Forzani.
} 
avec son écriture béhavioriste qui en devient le personnage phare. Le néopolar et son prolongement le roman noir s'installent en France quelque peu après la dissolution des idéaux de Mai 68. Les genres nargués par la critique et le lectorat cultivés deviennent la plateforme idéologique de tout mouvement gauchiste déradicalisé avec la réussite du Parti socialiste sous la présidence de François Mitterrand lors des élections de 1981.

En ce qui concerne le point de vue sociologique sur la littérature Karel Krejčí, constate déjà en 1944 : «Au niveau idéologique, la littérature précède le changement social, mais l'image artistique de cette nouvelle réalité sociale se crée bien plus tard, au moment où la nouvelle classe est déjà bien constituée et qu'elle a eu suffisamment de temps pour créer son propre style. ${ }^{6}$ Cette constatation s'accorde sur ce point avec Barthes selon qui en 1953 « chaque régime possède son écriture, dont l'histoire reste encore à faire. $»^{7}$

Pourtant, la spécificité du polar et plus précisément du polar français et de la littérature noire qui en dérive ensuite et qui d'une façon indirecte décrit les changements socio-politiques de la société française de la fin des Trente glorieuses et des crises des années 1970-1990 consiste dans le fait qu'il ne cherche pas à se distinguer au niveau artistique et se retranche délibérément en marge du champ littéraire pour pouvoir y continuer son action politique. ${ }^{8}$

En ce qui concerne Manchette, il est bien conscient du fait que la réalité du polar américain des années 1920-1940 dont il s'inspire est bien sûr déjà dépassée, mais il est persuadé que : « Avant de produire le polar, le triomphe de la contre-révolution en Amérique a produit la réalité dont le polar parle. » ${ }^{9}$ "La contre-révolution » qui est en Europe d'après-guerre prise pour le triomphe de la lutte politique des parties de la social-démocratie signifie

\footnotetext{
${ }^{6}$ Karel KREJČÍ, Sociologie literatury, Grada, Praha, 2008, p. 85. « Literatura totiž ideologicky obvykle sociální převrat předchází, avšak $\mathrm{k}$ uměleckému ztvárnění nové sociální skutečnosti dochází až značně později, kdy nová třída se plně konstituovala a měla dosti času, aby si vytvořila svůj styl vlastní. » Karel Krejčí (1904-1979).

${ }^{7}$ Roland BARTHES, Le Degré zéro de l'écriture suivi de Nouveaux essais critiques, Seuil, Paris, 1972, p. 22.

8 ..., nous avons constaté $[\ldots]$ que, constamment décliné de la part de la critique littéraire, le genre noir s'est au cours des années 1970-1990 transformé plutôt en outil de critique sociale et que son exclusion des structures " officielles » servait à ses connaisseurs de confirmation de la bonne direction de leur raisonnement désapprobateur de la situation politique et sociale de l'époque. Ainsi à la fin des années 1990, [...] le milieu du genre noir s'était situé plus dans le champ idéologique (de gauche) que dans les champs médiatique ou paralittéraire ou il était renvoyé constamment. Marcela POUČOVÁ, Sous les pavés le noir! Le roman noir dans la France post-68, Muni press, Brno, 2017, p. 10.

${ }^{9}$ Jean-Patrick MANCHETTE, Chroniques, Payot \& Rivages, Paris, 2013, p. 80.
} 
alors pour Manchette et ses successeurs surtout la dictature du capital dont l'effet influence profondément la vie des citoyens et la rend toute aussi contrerévolutionnaire.

La constatation de Manchette que « le polar est histoire de la criminalité et du gangstérisme, c'est-à-dire histoire de la violence obligée des pauvres après la victoire du capital ${ }^{10}$ peut très bien se comprendre dans le cadre des Etats-Unis des années du capitalisme sauvage, mais pas en ce qui concerne la France des années 1970. Les grandes crises économiques de l'époque font bien sûr un décor digne du genre noir, mais au lieu de la criminalité et du gangstérisme des années 1950 elles font naître le terrorisme de gauche et de droite radicales qui est en tant que tel dénoncé par la société majoritaire. Manchette a beau expliquer que « s'il n'y a plus de bonne fin possible, tous les moyens sont mauvais $»^{11}$, l'action terroriste dans la France ${ }^{12}$ des années 1970$1980^{13}$ est une suite d'actes de groupuscules à orientation politique extrémiste pourvue de raisonnements abstrus.

L'étude d'un tel groupuscule apporte le roman de Manchette peut-être le plus réussi Nada. Manchette sort le roman en 1973. La période « des années de plomb » suit son cours depuis quelques années mais personne ne sait qu'il s'agira d'une aussi longue période (une vingtaine d'années). Manchette ne peut se douter non plus que l'impact dévastateur de toute l'action terroriste sur la société européenne se finalisera dans - si on reste dans le lexique de Manchette - le plus grand triomphe de la contre-révolution. Ne sachant rien de cela et malgré ses convictions politiques, l'écrivain n'est pas dupe et lance au lecteur un message catégorique : "Le terrorisme gauchiste et le terrorisme étatique, quoique leurs mobiles soient incomparables, [...] sont les deux mâchoires du même piège à cons. $»^{14}$

\footnotetext{
${ }^{10}$ Ibid., p. 82.

${ }^{11}$ Ibid., p. 81.

${ }^{12}$ Les origines de l'action terroriste issue du milieu étudiant qui envahie l'Europe et notamment l'Allemagne à la fin des années 1960 et persiste jusqu'à la fin des années 1980 se trouvent dans la contestation de la guerre du Vietnam et le conflit entre Israël et la Palestine et se distinguent ainsi de l'action terroriste française de droite des années 1960 (OAS) et de son caractère mitigé dans les années 1970-1980. Le terrorisme de gauche français des années 1970-1980, quant à lui, est l'héritier direct du mouvement anarchiste né à la moitié du XIX ${ }^{\mathrm{e}}$ siècle qui coexiste depuis toujours avec la lutte ouvrière sans la dominer pourtant.

${ }^{13}$ Voir par exemple : Chronologie des actes terroristes en France. (2018, avril 8). Wikipédia, l'encyclopédie libre. Page consultée le $9: 58$, avril 8, 2018 à partir de https://fr.wikipedia.org/wiki/ Chronologie_des_actes_terroristes_en_France\#Ann\%C3\%A9es_1970.

${ }^{14}$ Jean-Patrick MANCHETTE, Nada, Gallimard, Paris, 1999, p. 215.
} 
La lecture historique du roman s'avère alors nécessaire pour que le décalage temporel n'obscurcisse pas son message. Le roman décrit l'histoire du kidnapping de l'ambassadeur américain effectué en plein Paris par un groupe de terroristes de gauche du nom de Nada. Manchette présente successivement les protagonistes principaux : Buenaventura Diaz dit le Catalan, Benoît D'Arcy, Marcel Treuffais, Nathan Mayer, André Epaulard et Véronique Cash. Sept personnes aux âges et aux destins différents qui se rassemblent pour planifier et effectuer le kidnapping.

L'écriture béhavioriste de Manchette les présente brièvement mais de manière significative. Les membres du groupe Nada sont des personnages sans passé ou au contraire avec un passé assez chargé. Parmi eux, seul Epaulard a droit à une description plus détaillé de son parcours que Manchette place entre 1940 et 1960 et lui donne un passé de contrebandier, maquis, tueur à gage et membre des corps révolutionnaires dans les pays du tiers monde un peu partout sur le globe. De cette époque il connait le Catalan et D'Arcy dont les vies se déclinent dans des circonstances semblables. Ce trio n'a plus d'idéaux, lassés de la vie, ils cherchent à justifier leur existence et subvenir à leurs dépenses. Par cet acte désespéré, seul le Catalan croit pouvoir changer quelque chose, Epaulard se décide à participer au coup par un simple : «Eh, merde, quoi !»

Quant aux « jeunes », Mayer (garçon dans un restaurant), Treuffais (jeune professeur de philo) et Cash (qui se proclame prostituée), leurs métiers sont tout aussi différents que leurs opinions politiques - floues se coupant dans le mépris contre l'establishment. Cash, comme souvent chez Manchette s'il s'agit d'une femme, s'exprime sur ce sujet par un propos exalté :

Je suis pour l'harmonie universelle, dit-elle, et pour la fin pitoyable d'Etat civilisé. Sous mon apparence froide et apprêté se cachent et bouillonnent les flammes de la haine la plus brûlante à l'égard du capitalisme technobureaucratique qu'a le con en forme d'urne et la gueule en forme de bite. ${ }^{15}$

De ce trio-ci, c'est seulement Treuffais qui est présenté en détail. Le personnage d'un prof de philo dans un lycée huppé ne laisse pas douter un lecteur averti qu'il s'agit du portrait ironique de l'auteur en personne. Et tout comme lui dans son écriture, Treuffais prend finalement la distance de l'acte terroriste :

${ }^{15}$ Ibid., p. 66. 
«Bordel, Buen, c'est parce que je suis communiste libertaire que je vous demande de suspendre l'opération. [...] Tu sombres dans le terrorisme et ça, c'est con. Le terrorisme ne se justifie que dans une situation où les révolutionnaires n'ont pas d'autre moyen de s'exprimer et où la population soutient les terroristes. ${ }^{16}$

Dans la partie des défenseurs de la loi toute aussi dépourvue de sympathie ou d'antipathie, Manchette poursuit son écriture béhavioriste et garde une impartialité flaubertienne dans la description des petites et grosses lâchetés des caractères de ses personnages et de leur résignation à une vie dénuée de sens. Tel est surtout le personnage de l'inspecteur de police Goémond qui - en tant que roue dans l'engrenage de la machine étatique - ne dispose que de très peu de responsabilités, ce qui fait de lui un monstre redoutable parce que non coupable. En même temps, il est un des rares, et peut-être le seul, acteur du drame à travers lequel le lecteur peut se rendre compte du fonctionnement du mécanisme qui tire les ficelles : l'Etat se proclamant gardien de l'ordre et de l'ensemble des valeurs humaines et se croit qualifié pour éliminer toutes sortes de marginalité considérées comme dangereuses. Ce point de vue que lecteur peut comprendre seulement de façon rétrograde, Manchette l'explique sur la première page de son roman dans la lettre qu'un jeune gendarme participant à l'extermination brutale du groupe adresse à sa mère :

Sérieusement, petite Maman, tu voudrais d'un pays sans police ? ... Je ne dis pas qu'il n'y a pas une majorité de bonnes gens au bourg mais toutefois, rien que dans notre paisible communauté rurale, s'il n'était pas su qu'il y a une police et prête à tirer au besoin, j'en vois déjà qui n'hésiteraient pas, sans parler des romanichels. ${ }^{17}$

Franc Frommer appelle ce procédé « une mise en situation, une forme de pré-récit allégorique résumant la fable qui va suivre. ${ }^{18}$

La marginalité des personnages du roman s'exprime tout d'abord au niveau intérieur. Avant de passer à l'acte, les protagonistes ne présentent aucun signe de marginalité extérieure. D'ailleurs, Mayer et Treuffais ont même un emploi stable tout comme le commissaire Goémond. La marginalité des terroristes est ancrée dans leur orientation politique d'extrême gauche déroutée.

\footnotetext{
${ }^{16}$ Ibid., p. 126-127.

${ }^{17}$ Ibid., p. 12.

${ }^{18}$ Franck FROMMER, Jean-Patrick Manchette, Le récit d'un engagement manqué, Kimé, Paris, 2003, p. 37.
} 
Tandis qu'ils imaginent accomplir un fait d'armes, le reste de la société, y compris leurs frères spirituels des différentes fractions de la gauche militante s'en distancient publiquement. Pour marquer cette distance de façon nette, Manchette utilise la technique des coupures de journaux de Dos Passos : les manchettes désapprobatrices imprimées en différents caractères forment tout le chapitre 15 ( « LES FORCES OUVRIÈRES ET DÉMOCRATIQUES SE PRÉPARENT À UNE VIGOUREUSE RIPOSTE CONTRE LES PROVOCATEURS. $»^{19}$ ). Pour une distance encore plus marquée, l'auteur ne fait ensuite que citer de la presse les revendications du groupe sans donner au lecteur la possibilité de prendre connaissance textuellement du manifeste en question. Il lui transmet seulement l'opinion des journaux comme celui du Monde : « le style en est ordurier [...] et la puérilité de certaines affirmations, d'un anarchisme archaïque et sans mélange, prêterait à sourire en d'autres circonstances. $»^{20}$

Si les terroristes affirment leur raison de passer leur l'existence en marge et Treuffais opte pour une marginalité intellectuelle (on ne sait pas si c'est par lâcheté ou pour une réelle conviction politique), le commissaire Goémond trouve sa place dans la machinerie étatique et il y est protégé de toutes intempéries :

- Oui, dit le chef de cabinet. S'ils le liquidaient pendant l'assaut, quelle horreur ! Il arrive qu'une petite frange de l'opinion publique éprouve pour l'extrême gauche une sympathie irréfléchie, mais cette sympathie n'est plus possible lorsque les gauchistes révèlent leur vraie nature en assassinant froidement un prisonnier sans défense. ${ }^{21}$

Mais au moment où la roue commence à gêner et empêche le bon fonctionnement de l'engrenage, Goémond est éliminé du système et se trouve en marge, tout comme les victimes de sa haine. C'est ainsi qu'il se fait renvoyer par le même chef de cabinet :

- Vous vous croyez un peu trop, commissaire, depuis quelque temps. Vous croyez peut-être que vous êtes au-dessus des lois ? Vous avez mené cette opération avec une brutalité qui ne peut pas être tolérée et qui ne le sera pas. ${ }^{22}$

\footnotetext{
${ }^{19}$ Jean-Patrick MANCHETTE, Nada, Gallimard, Paris, 1999, p. 108.

${ }^{20}$ Ibid., p. 124.

${ }^{21}$ Ibid., p. 166.

${ }^{22}$ Jean-Patrick MANCHETTE, Nada, op. cit., 1999, p. 219.
} 
C'est ici où la phrase de Manchette : «Quand la contre-révolution a triomphé, c'est toute la vie humaine qui devient contre-révolutionnaire. ${ }^{23}$, révèle son principe et explique le fondement du nihilisme omniprésent que le roman met en scène : dans la société moderne l'homme est réduit à une unité de consommation qui, au moment où elle refuse de consommer de la façon prescrite ou que sa propre production devient désuète ou indésirable, doit être, tout comme chez Huxley, mise hors circuit. La marginalité intérieure voire intellectuelle tout comme extérieure (l'action terroriste) chez Manchette ne représente pas une forme de négation libératrice et n'a aucune valeur ajoutée. Dans le monde de Manchette on est prédestiné à un échec dès le moment où on s'éloigne du troupeau peu importe la façon.

Cette constatation aussi froide que tranchante est soulignée par le style de Manchette. Sa narration, dépourvue de tout sentiment, se veut objective voire tout aussi neutre que possible. Il semble pourtant faux d'attribuer au style de Manchette l'exactitude d'une transcription cinématographique qui bannit l'introspection psychologique. Le film, à part la description du milieu et la présentation des dialogues, fait comprendre le développement psychologique des personnages par le travail de corps et de visages des acteurs. Rien de tel chez Manchette. Plus le lecteur en apprend sur le décor, moins il en apprend sur les personnages. Les dialogues manquent même de verbes d'introduction qui pourraient signaler la disposition psychique des locuteurs. Cette technique fautivement interprétée documentaliste (lettre du gendarme, titres des journaux, mais aussi descriptions détaillées n'ayant aucun rapport au sujet : « Dans l'armoire, deux slips, une paire de draps, six paires de chaussettes de fil, deux cravates unies, deux chemises en nylon et un pardessus en poil de chameau vieux de dix ans. $\gg^{24}$ et en même temps une absence quasi-totale d'informations concernant le propriétaire des affaires énumérées) laisse lecteur perplexe, face à un vide existentiel. Elle le force de se poser des questions : qu'est-ce qu'on apprendrait de cette façon sur moi ? Qui suis-je au regard des autres et quelles sont mes convictions? Sont-elles aussi lamentables que celles des personnages du roman ? Ne suis-je moi aussi seulement qu'un paumé en marge ? L'engagement de l'auteur se trouve alors tout aussi dans la forme que dans le sujet. C'est le style de Manchette qui, en empêchant le lecteur de s'identifier aux personnages, lui présente le miroir. L'absence de prise de position force le lecteur à en prendre une et cela lui fait mal.

\footnotetext{
${ }^{23}$ Jean-Patrick MANCHETTE, Chroniques, Payot \& Rivages, Paris, 2013, p. 81.

${ }^{24}$ Jean-Patrick MANCHETTE, Nada, Gallimard, op. cit., p. 36.
} 
Une autre sorte de marginalité est présente dans le dernier roman achevé de Manchette La position du tireur couché sorti en 1981. Si Nada travaille la thématique des activistes politiques militants, ce qui est, pendant les années de plomb, la problématique touchant la population française au quotidien, $L a$ position du tireur couché traite le sujet du crime organisé lié aux structures étatiques. Ceci, tout aussi existant, est pourtant beaucoup moins visible. Les structures et le comportement de ce genre de criminalité sont bien sûr moins connus du grand public et ceci permet à Manchette de jouer plus avec les procédés du roman hard-boiled classique. De fait, le roman est construit comme une série de tueries spectaculaires dont le déroulement rapide tient le lecteur en haleine et dont le dénouement inattendu laisse dans la conscience du lecteur un goût d'amertume d'être dupe le long de l'histoire tout comme dans la vie réelle.

Martin Terrier, proche de la trentaine, est un tueur à gages. Ce métier, il avait adopté à la fin de sa carrière de mercenaire. Un personnage de cette catégorie peut sembler déjà bien marginal. Si la société humaine accepte la guerre, elle n'accepte en aucun cas le meurtre d'autrui et l'importance de cet interdit est déjà dans les Dix commandements. Un tueur à gages est porteur d'une marginalité double : tueur et en plus sans rapport émotionnel avec la victime. En créant un tel personnage principal, Manchette crée et joue ensuite encore avec un désaccord dans la perception du lecteur qui a une tendance naturelle à sympathiser avec le héros principal. Cette déstabilisation est encore renforcée par l'écriture béhavioriste qui l'empêche de se mettre dans la peau des personnages. Bien que Manchette ne soit plus aussi strict que dans Nada et propose une description plus détaillée du comportement de ses personnages, il reste toujours assez réservé et ses notes touchant le monde intérieur de ses héros ressemblent plutôt aux didascalies d'un scénario :

[ ... ] Je voulais me rapprocher de cette espèce de grande fourchette. (Elle frissonna.) Il fallait que quelqu'un fasse quelque chose. Ils nous auraient tués, hein ? (Elle fronça les sourcils. Son regard ne semblait plus vide de pensée comme naguère ; au contraire il était sérieux, elle paraissait se concentrer.) Je n'ai jamais vu des êtres pareils, observa-t-elle. Mais tu es comme eux ? Ou bien non ? (Soudain sa voix et son regard étaient redevenus incertains.)

- Je suis comme eux. Pas seulement. Mais je suis comme eux. 
- Eux non plus n'étaient pas seulement comme ça, je suppose, dit Anne. (Elle eut un gloussement de pure nervosité :) C'est très philosophique, ce que je viens de dire, commenta-t-elle. ${ }^{25}$

A travers ce dialogue, on peut quand même constater que Terrier dans sa phrase sèche exprime tout son univers. Etre un assassin est pour lui un métier comme tous les autres et il lui sert à gagner sa vie. Il n'éprouve pas de haine envers ses adversaires, ils sont pour lui des concurrents dans son secteur économique à une exception près, perdre l'affaire signifie perde non seulement de l'argent mais aussi la vie. Si son métier fait de Terrier quelqu'un en marge, à travers la lecture on apprend la raison du choix de la profession qui a, elle aussi, liée avec la marginalité - cette fois sociale. Martin, fils de parents pauvres, pendant ses études au lycée, rivalise avec ses copains de classe provenant du milieu bourgeois. Il tombe amoureux d'Anne venant d'une famille plus qu'aisée. Mais le père d'Anne lui laisse entendre qu'il n'est pas le bienvenu. Ce mépris touche profondément Martin qui jure à Anne de revenir dans dix ans, après avoir acquis suffisamment d'argent pour pouvoir l'épouser. Un rêve puéril, une réaction par bravade, certes mais surtout un manque réel de sens moral : pour réussir sa résolution, Terrier devient un excellent tueur à gages sans scrupules et sans émotions.

Le début de l'histoire trouve Terrier au moment où il pense rassembler suffisamment d'argent pour pouvoir épouser Anne. Cette décision peut rétroactivement faire comprendre au lecteur un certain manque d'intelligence émotionnelle du héros qui poursuit son but avec obstination, horizon borné et sans scrupules. La décision de quitter les services du mystérieux M. Cox s'avère néanmoins dangereuse. La « société » ne prévoie pas que ses employés puissent la quitter à leur gré. Pratiquement dès l'instant de sa démission, Terrier est poursuivie par les agents de différentes organisations qui veulent tous sa peau. Sa marginalité se décuple ainsi.

Le personnage de Terrier se retrouve dans une situation semblable à celle de l'inspecteur Goémond. Si le personnage du commissaire Goémond offre l'image d'un homme de pouvoir corrompu, le personnage de Terrier retrouve certains éléments caractéristiques de Julien Sorel de Stendhal, un jeune homme prometteur de condition modeste aux ambitions immodérées et un sens de la moralité minimisé. Les deux personnages représentent pourtant deux faces d'une même médaille : étant membres d'une structure « officielle » et poursuivant diligemment ses instructions, ils bénéficient de sa protection.

\footnotetext{
${ }^{25}$ Jean-Patrick MANCHETTE, La position du tireur couché, Gallimard, Paris, 1988, p. 90-91.
} 
Au moment où ils deviennent gênants, ils sont mis hors circuit et on cherche (chacun à sa façon) à les éliminer. Leur marginalité s'avère destructive et dangereuse non seulement pour la société (ils sont d'ailleurs payés pour être comme cela) mais avant tout pour eux-mêmes. Il n'est pas étonnant que Manchette fait finalement découvrir au lecteur que la fameuse «société » qui employait Terrier n'était rien d'autre que les services secrets de la République française. Ces derniers, pendant des années, abusaient de lui pour se débarrasser des bonzes des groupes terroristes à travers le continent tout en renvoyant la responsabilité au camp des adversaires idéologiques - les Soviétiques. Ici, nous nous retrouvons bien dans l'univers de Manchette pour lequel la fiction noire sert de champ de bataille idéologique. Dans ces chroniques il constate d'ailleurs :

L'idéologie dominante ne veut pas qu'on se demande si, des fois par hasard, dictature totalitaire et démocratie représentative ne seraient pas deux œufs du même panier, deux mères du même merdier historique, alternant gaiment, s'engendrant joyeusement l'une à l'autre, pour prolonger la domination du capital. ${ }^{26}$

Ainsi le pouvoir politique sacrifie Goémond pour faire taire la rue ( «On a crié votre nom dans les rues de Paris, cet après-midi $[\ldots] »^{27}$ ). Et Terrier, devenu incontrôlable, est présenté au public comme quelqu'un qui « avait été formé par le KGB dans son école spéciale d'Odessa, puis dans les camps palestiniens et auprès de la DGI cubaine. $»^{28}$

Le troisième roman choisi Le petit bleu de la côte Ouest et publié dans la Série noire en 1976. La notion de la marginalité sur laquelle repose la trame narrative des deux romans précédents n'est que très peu prononcée et son rôle est bien différent. Manchette utilise cette fois-ci surtout le côté satirique de son écriture et de plus, il truffe son histoire d'allusions littéraires, musicales, culturelles et politiques de son actualité.

Manchette caractérise son Georges Gerfaut, le héros principal du livre, comme un homme en pleine crise du deuxième âge dont la vie confortable d'un cadre moyen et père d'une jolie petite famille ne satisfait plus. Témoin d'un assassinat passé pour un accident de la route, il devient gênant et deux tueurs sont envoyés pour l'éliminer. Au lieu de se faire tranquillement assassiner,

\footnotetext{
${ }^{26}$ Jean-Patrick MANCHETTE, Chroniques, Payot \& Rivages, Paris, 2013, p. 80.

${ }^{27}$ Jean-Patrick MANCHETTE, Nada, Gallimard, Paris, 1999, p. 218.

${ }^{28}$ Jean-Patrick MANCHETTE, La position du tireur couché, Gallimard, Paris, 1988, p. 165.
} 
Gerfaut sans prévenir la police, se met à la contre-attaque et finalement il se sauve pour mener une vie tranquille dans une profonde campagne. Après plusieurs mois, insatisfait de son évasion et après avoir bouclé l'affaire (Gerfaut tue les deux assassins et l'homme qui avait commandé le meurtre initial), le héros, revient à la maison et simule l'amnésie.

Dès le début du livre Manchette se moque systématiquement de ses personnages en attirant l'attention sur leurs vies médiocres. Gerfaut, avant de devenir la proie des assassins, déteste les vacances familiales en Aquitaine, trouve sa belle-mère «conne » et selon la situation, il «picole » de façon plus ou moins modérée. Les deux tueurs passent leur temps libre (c'est-à-dire entre les meurtres) à faire des exercices isométriques. Leur client, ancien officier de l'armée dominicaine - un tortionnaire redoutable, réfugié en France, époussette constamment sa chaîne haute-fidélité et écris des textes sur le fonctionnement de la démocratie.

Manchette trouvait que son livre était plutôt une moquerie qu'un témoignage social. ${ }^{29}$ Mais celle-ci remise dans le contexte des crises économiques et politiques de l'époque est pourtant plus que mordante.

L'originalité de l'histoire de Gerfaut consiste dans le fait que son déroulement délirant ne retrace en effet que des bribes d'un quotidien le plus banal et que la marginalité des personnages n'est qu'apparente : en dehors du fait d'avoir assassiné de quelques à des dizaines de personnes, ils se croient tous réguliers et dans d'autres circonstances ils seraient même à plaindre. Le style d'écriture béhavioriste de Manchette souligne encore une fois la monstruosité du comportement amoral de ses personnages qui dénués de scrupules ne se consacrent et ne s'intéressent qu'à eux-mêmes.

«L'image qu'il avait de lui-même s'inspirait d'un roman policier lu dix ans auparavant, et d'un petit western baroque et métaphysique vu l'automne précédent au cinéma Olympic. ${ }^{30}$ Tel est par exemple la caractéristique ironique de Gerfaut qu'il se donne après avoir tué son adversaire et échappé à sa propre mort. L'officier de l'armée Alonso - un tueur de masse se croit en droit d'écrire : «La meilleure manière de mettre la fin à la violence, c'est de punir les individus qui s'y livrent, quelle que soit leur catégorie sociale. $»^{31}$

Si pour Manchette son écriture signifie avant tout une critique et une désignation des excès de l'idéologie régnante, l'analyse de ses personnages

\footnotetext{
${ }^{29}$ Voir Jean-Patrick Manchette au sujet de "Le petit bleu de la côte ouest", Apostrophes de Bernard Pivot, Antenne 2, 20 juillet 1979, consulté le 17:58 avril 24, à partir de http://www.ina.fr/video/ I04328892.

${ }^{30}$ Jean-Patrick MANCHETTE, Le petit bleu de la côte Ouest, Gallimard, Paris, 1966, p. 91.

${ }^{31}$ Ibid., p. 172.
} 
montre des racines plus profondes de leur marginalité. Manchette peut-être inconsciemment mais d'une façon très significative illustre ce que Jung dans son essaie Dialectique du Moi et de l'inconscient explique sur l'individu et la collectivité :

Dès lors, naturellement, seuls prospéreront la société et ce qu'il y a de collectif dans l'individu. Tout ce qu'il y a d'individuel en lui est condamné à sombrer, c'est-à-dire à être refoulé. De ce fait tous les facteurs individuels deviendront inconscients, tomberont dans l'inconscient; ils y végéteront et s'y transformeront selon une loi implacable en une manière de négativité systématique, de malignité principielle, qui se manifestent en impulsions destructrices et en comportements anarchiques. Ces tendances deviendront agissantes sur le plan social, chez l'individu tout d'abord : certains sujets à tempérament prophétique deviennent l'instrument de crimes à sensation (meurtre de roi, etc.); mais elles se font sentir chez tous de la façon indirecte, à l'arrière-plan, par une décadence morale inévitable de la société. ${ }^{32}$

Pris de ce point de vue, le geste désespéré des anarchistes du groupe Nada, le comportement dérouté puisque impunissable des représentants d'Etat (Goémond, Alonso) et l'insuffisance morale de Terrier et Gerfaut ne font que témoigner la décadence morale de la société moderne décrite par Jung déjà dans les années 1930. Ce dernier explique la cause de la perte de responsabilité de l'individu par la relativisation de son rôle dans la machinerie étatique. Manchette quant à lui ne fait que témoigner une quarantaine d'années plus tard la déshumanisation de la société moderne dans laquelle la morale est remplacée par l'esprit moutonnier des individus privés de la liberté de disposer d'eux-mêmes. La lecture attentionnée de la marginalité des personnages de Manchette ne fait alors que s'accorder avec Jung sur le fait que : " Hors la liberté, point de moralité. $»^{33}$

\footnotetext{
${ }^{32}$ Carl Gustav JUNG, Dialectique du Moi et de l'inconscient, Gallimard, Paris, 2013, p. 74.

${ }^{33}$ Ibid., p. 75.
} 